\title{
Socio-economic Inequality in Stunting among Children Aged 6-59 Months in a Ugandan Population Based Cross-sectional Study
}

\author{
Baru Ruth Sharon Apio, Ratib Mawa*, Stephen Lawoko, Krishna Nand Sharma \\ Department of Public Health, Faculty of Health Sciences, Victoria University Kampala, Kampala, Uganda \\ Email address: \\ Sharon2ruth@gmail.com (B. R. S. Apio), ratib@vu.ac.ug (R. Mawa), deanhealthscience@vu.ac.ug (S. Lawoko), \\ vicechancellor@vu.ac.ug (K. N. Sharma) \\ ${ }^{*}$ Corresponding author
}

\section{To cite this article:}

Baru Ruth Sharon Apio, Ratib Mawa, Stephen Lawoko, Krishna Nand Sharma. Socio-economic Inequality in Stunting among Children Aged 6-59 Months in a Ugandan Population Based Cross-sectional Study American Journal of Pediatrics Vol. 5, No. 3, 2019 , pp. 125-132.

doi: 10.11648/j.ajp.20190503.18

Received: April 18, 2019; Accepted: June 14, 2019; Published: August 6, 2019

\begin{abstract}
Socio-economic status is an important predictor of stunting, however published population based studies on socioeconomic inequalities in stunting among children under-five years of age is scarce in Uganda. Data from the 2016 Uganda Demographic and Health Survey was used to identify possible socio-economic inequalities in stunting among 3941 children aged 6-59 months. Multivariate binary logistic regression models were fitted to calculate the odds ratios and their corresponding $95 \%$ confidence intervals for stunting by maternal formal education and household wealth index. The overall prevalence of stunting among children was $30.1 \%$. The risk of stunting was higher among children whose mothers had no formal education (OR: 4.35; 95\% CI, 2.45-7.71), attained primary (OR: 2.74 95\% CI, 1.62-4.63) and secondary level education (OR: $2.3095 \%$ CI, 1.34-3.96) compared to those whose mothers attained tertiary level education. Similarly higher risk of stunting was found among children that lived in the poorest (OR: $1.7895 \% \mathrm{CI}, 1.23-2.59)$, poorer (OR: 1.88; 95\% CI, (1.282.74), middle (OR: 1.91, 95\% CI, 1.31-2.77) and richer households (OR: 1.60; 95\% CI, 1.10-2.32) compared to those in the richest households. Socio-economic differences in stunting among children under-five years of age were found. Targeting stunting prevention interventions to less affluent mother-child dyads and households might be important in reducing social inequalities in stunting among children under-five years of age in Uganda.
\end{abstract}

Keywords: Stunting, Children, Socio-economic Status, Inequalities, Uganda

\section{Introduction}

The deleterious effect of stunting on children's physical and cognitive development and later life risk of chronic diseases is well established [1-4]. This explains the concerted global efforts to reduce the burden of stunting among children under-five years of age. However across the globe, there has been slow decline in the prevalence of stunting among children under-five years of age over the last two decades [5-7]. Recent joint United Nation Children`s Fund (UNICEF), World Health Organization (WHO) and World Bank estimates showed that globally, $21.9 \%$ of children under-five years of age were stunted, suggesting a $10.6 \%$ decline from the year 2000 [7]. However in the same period,
Africa remained the only region where the number of cases of stunting increased from 50.3 to 58.8 million from the year 2000 to 2018 respectively $[6,7]$. This increasing trend in the prevalence of stunting among children under-five years of age has serious implications for socio-economic development of the continent. Although Uganda recorded a steady decline in the prevalence of stunting among children under-five years of age in the last three decades [8], the number of affected children remained high (2.2 million children) approximately $29 \%$ [9]. This prevalence of stunting among Ugandan children aged 6-59 months remains unacceptably high when compared to global standards. The reversal of this declining trend in the prevalence of stunting is probable, given the recent increase in level of poverty in the country [10], poor 
infant and young child feeding practices and limited access to potable water, appropriate hygiene and sanitation practices nation-wide [11]. If unaddressed, this might impede the achievement of the sustainable development goals especially on child health in Uganda [11, 12]. To achieve the WHO target of reducing the prevalence of stunting among children under-five years of age by $40 \%$ by the year 2025 [13], it's therefore important to target stunting prevention interventions to high risk groups.

Socio-economic status is an established social determinant of health [14]. The context, causal pathways, and the consequences of stunting among children under-five years of age are multi-faceted and well-established as published in the WHO conceptual framework for stunting among children under-five years of age [3]. Most determinants of stunting among children under-five years of age are linked to socioeconomic status at individual and household level and formal knowledge on child care and feeding practices. Studies conducted in Sub-Saharan Africa showed consensus on existence of household socio-economic inequalities in stunting among children under-five years of age [15-18] even though there are variations in the setting, study populations and measures of household socio-economic status that obscure direct comparison of the findings.

When maternal education level was used as a proxy measure of socio-economic status at individual level, most studies found a decreasing trend in the risk of stunting among children under-five years of age by increasing level of maternal formal education. A South African birth cohort study conducted in the metropolitan area of SowetoJohannesburg found an association between maternal education level and stunting among children under-five years of age [19]. On the same note, a Ugandan cross-sectional study based on the 2011 UDHS found an increasing risk of stunting by decreasing level of maternal education. Children whose mothers had no formal education were 1.47 times more likely to be stunted compared to those whose mothers had tertiary level educations [20]. Similarly in most other studies conducted in sub-Saharan Africa, demonstrated an inverse relationship between maternal education level and stunting among children under-five years of age [18, 21, 22], implying maternal educational differences in stunting among children under-five years of age are wide-spread in subSaharan Africa. The pathways through which maternal education provides protective effect against stunting among children under-five years of age linked to the health and nutrition knowledge of educated mothers and the economic benefits derived from education, however the economic benefits of education to individuals in developing countries has been challenged by other scholars hence the validity of education as a proxy measure of socio-economic status in developing countries questioned [23]. This argument seems to resonate well with evidence from a meta-analysis of studies conducted in low and middle income countries which found modest effect of education on the risk of stunting among children under-five years of age [24]. Nevertheless challenges still persist in use of income as a measure of socio-economic status at individual level, making it unlikely to avoid use of education level as a proxy measure of socioeconomic status.

Published population based research articles on socioeconomic inequalities in stunting among children under-five years of age are scarce in Uganda. The most recent publication based on 2011 UDHS revealed socio-economic patterning in the risk of stunting among children under-five years of age [20]. Other studies were conducted were conducted over a decade back and in localized settings (districts or hospitals [25, 26] limiting generalization of the results to all children underfive years of age in Uganda. Other than socio-economic status, several other factors have been shown to influence occurrence of stunting among children under-five years of age. Studies conducted in sub-Saharan Africa found an increasing trend in the risk of stunting by age among children under-five years of age [16, 17, 25-27]. Gender differences in the risk of stunting have also been reported in studies conducted in sub-Saharan Africa. A systematic review and meta-analysis study found higher risk of stunting among boys than girls [28]. Other related studies conducted in Uganda and Gambia also found similar gender disparity in the risk of stunting among children under-five years hence boys are seem to be a high risk group for stunting when compared to girls [20, 25, 29]. However the cause of this gender disparity is not clear although other studies suggested that young boys often tend to be more active and stay away from home while girls are always at home with their mothers giving them the opportunity to eat any-time as their mothers prepare meals as such boys tend to fall sick more frequent than girls [30]. Mother-child dyad socio-demographic characteristics found to be associated with stunting among children under-five years of age include maternal marital status [31], number of children in the household, maternal occupation, duration of breastfeeding and bottle feeding [32]. However discrepancies were reported with regards to the magnitude and the direction of the association between maternal marital status and stunting among children under-five years of age [27]. In addition to the above Maternal age below 24 and above 35 years, short birth intervals, low birth weight, low maternal body mass index were also found to be associated with stunting among children under-five years of age [15]. This study was conducted to provide results that will add into the limited knowledge base on socio-economic inequalities in stunting among children under-five years of age in Uganda, a developing country with volatile economic environment, increasing socio-economic inequality, limited access to nutrition specific interventions and poor healthcare services. The aim is to determine socio-economic inequalities in stunting among children under-five years of age at individual and household level.

\section{Methods and Materials}

\subsection{Setting and Materials}

This is a nation-wide study conducted in Uganda. Uganda is a developing country located in East Africa. It has a weak 
healthcare system operated as public, private for profit and private not for profit. The materials for this study were obtained from the 2016 Uganda Demographic and Health Survey (UDHS) that was conducted between $20^{\text {th }}$ May and $16^{\text {th }}$ December 2016. The purpose of conducting the 2016 UDHS was to provide information on fertility and family planning, maternal mortality, child health, childhood mortality rates, domestic violence, HIV/AIDS. The survey respondents included all women aged 15-49 years and men aged 15-54 years in 1/3 of the selected households. A multistage cluster sampling was employed to select households and participates included in the survey. The Ugandan 2014 population and housing census sampling frame was used for the 2016 UDHS. It provided a complete list $(78,462)$ of census enumeration areas for the entire country. A census enumeration area is a village in rural areas and a block in urban areas in Uganda, a country divided into 112 administrative districts that are further sub-divided into subcounties, each sub-county is divided into parishes, and each parish into villages. The survey response rate for women was $91.34 \%$ in urban and $94.88 \%$ in rural areas and that of men was $81.74 \%$ in urban and $92.39 \%$ in rural areas. For more details on the sampling strategy employed in the 2016 UDHS, please refer to the final survey report [9]

\subsection{Study Design and Population}

This is a country wide cross-sectional study based on the 2016 UDHS. In children's dataset for the 2016 UDHS, a total of 15522 children were registered in the selected households. Of these children $812(5.2 \%)$ were deceased, 1586 (10.2\%) who didn't meet the criteria for anthropometric measurements, $19(0.1 \%)$ children with implausible height and weight measurements (flagged cases) and 9164 (59.0\%) who had either weight or height measurements missing were excluded, leaving a study population of 3941 children aged 6-59 months with valid anthropometric measurements.

\subsection{Outcome and Exposure Variables}

Stunting: The outcome investigated in this study is stunting among children under-five years of age, defined as height-for-age Z-score below negative two standard deviations (-2 SD) from the WHO Child Growth Standards median. Height was measured using Shorr Board ${ }^{\circledR}$ measuring boards and recorded to the nearest centimeter. Children younger than 24 months old were measured lying down on the board (recumbent length), while standing height was measured for those older than 24 months. Weight was measured with an electronic SECA 878 flat scale designed for mobile use. For very young children, the mother or caretaker was weighed first, and then later either the mother or the caretaker was re-weighed together with the child. An automatic two-in-one adjustment button allowed the mother's stored weight to be deducted and the baby's weight to be displayed on the scale (UDHS, 2016).

Socioeconomic status: Maternal education level and household wealth index were used as proxy measures of mother-child dyad socio-economic status at individual and household level. Validated 2016 UDHS questionnaire was used to collect the data on maternal education level and household wealth index. The household wealth index is a composite variable derived using the statistical method of the principle component analysis from a list of household properties that the women reported when asked to list assets and other properties the family had at the time of the survey. For more details readers can refer to the 2016 UDHS report and the principle component analysis methodology [11,33]. The index grouped mother-child dyads into five groups i.e. those living in the $20 \%$ poorest households, poorer, middle richer and richest households respectively. Self-reported maternal education level was assessed by asking the questions: 1) Have you ever attended school? 2) What is the highest level of school you attended? Mothers who responded "No" to the first question were categorized as having no formal education and those that responded to the $2^{\text {nd }}$ question were categorized as having attained: primary, "O" level, "A" level, and tertiary or university education level. Hence mothers were then grouped into four educational groups; no formal education, primary, secondary and tertiary level education.

\section{Sample size}

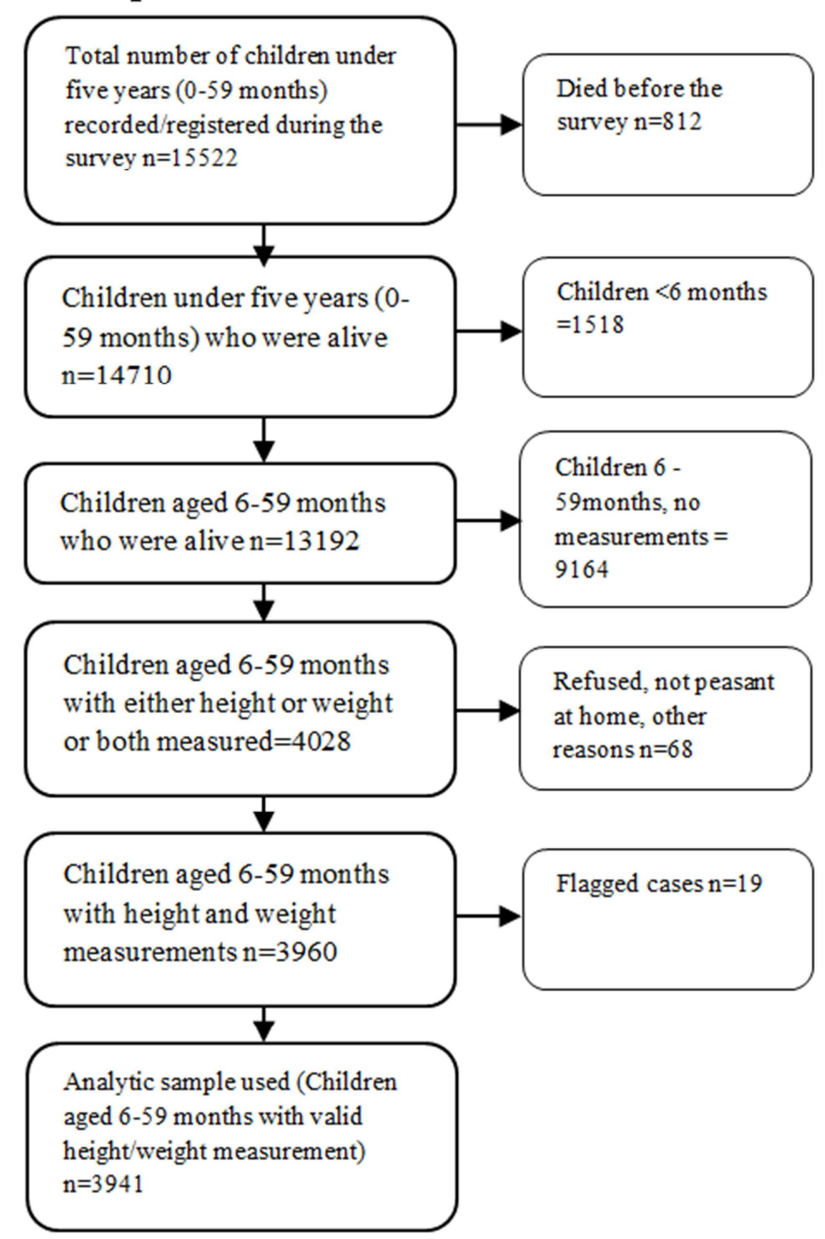

Figure 1. Schematic illustration of sample selection from the 2016 UDHS Children's data file. 


\subsection{Potential Confounders}

All potential confounding variables were either selfreported or measured using standardized equipment's and by trained survey enumerators. Maternal age was recorded as a continuous variable and later grouped into seven age groups of 15-19, 20-24, 25-29, 30-34, 35-39, 40-44, and 45-49. Maternal body mass index (BMI): A composite variable derived from measurement of maternal height in meters and weight in kilogram using calibrated height and weight measurement equipment. Mothers weight in kilograms was divided by their heights in meter squared to obtain their individual BMI. Mothers BMI were then categorized into underweight $(<18.5 \mathrm{~kg} / \mathrm{m} 2)$, normal $(18.5-24.99 \mathrm{~kg} / \mathrm{m} 2)$, overweight $(25-29.99 \mathrm{~kg} / \mathrm{m} 2)$, and $\geq 30 \mathrm{~kg} / \mathrm{m} 2)$. Maternal marital status was grouped into four categories: never married, married, widowed, living together, separated and divorced. Number of living children was recorded as a continuous variable; we then created 1-2, 3-4, and 5+ categories. Maternal tobacco use was recorded as a dichotomous variable, yes if the mother uses tobacco and no if she does not. Place of residence was also dichotomized into rural and urban. Child's age was recorded in months and categorized into 6-10, 11-15, 16-20, 21-25, 26-30, 31-35, 36$40,41-45,46-50,51-55$, and 56-59 months. Child sex was also a dichotomous variable recorded as 1 if male and 0 otherwise. Child's birthweight was recorded in kilograms and grouped into $<2500$ (low birth weight) and $\geq 2500 \mathrm{~kg}$ (average or above average birth weight). Child 's anemia status: was recorded as hemoglobin level $<8.0 \mathrm{~g} / \mathrm{dl}$ (anemic) and $\geq 8.0$ $\mathrm{g} / \mathrm{dl}$ (no anemia). Lastly the variable maternal health insurance was also recorded and used in the analysis as dichotomous variable; yes if the mother was insured and no if she had no insurance.

\subsection{Data Analysis}

Bivariate analysis was conducted to determine the distribution of children by their stunting status. Differences in the distribution of the prevalence of stunting among children under-five years of age across sub-categories of each categorical variable were tested using chi-square test. To calculate the odds ratios and their corresponding 95\% confidence interval for stunting by household wealth index and maternal education level, two binary logistic regression models were fitted adjusted for important confounding variables. Analysis was conducted using the statistical package for social scientists (SPSS version 24).

\subsection{Ethical Considerations}

Written permission was granted by the DHS data archivist for use of the 2016 UDHS secondary data. Before the survey data collection exercise, ethical approval of the survey was permitted by ICF International (Calverton, MD, USA) Institutional Review Board (IRB) and Uganda National Council of Science and Technology. Survey participants provided a written informed consent before the data collection exercise. Those that refused to participate in the survey were allowed to have their right of choosing not to participate in the study. Data for each child was recorded in anonymous manner hence each participant/child`s personal information was not identifiable.

\section{Results}

Table 1 shows the distribution of children by sociodemographic characteristics and the prevalence of stunting by various characteristics. There is uniform distribution of children by age with the lowest proportion of children $(6 \%)$ found in the age group of 56-59 months. Approximately half of the children were girls, majority had birth weights $\geq 2500$ grams, most were living with mothers who were either married or living with a partner. Most mothers were in the age ranges of 20-24, 25-29, 30-34, and 35-39 with the least proportion of children living with mothers in the age range of 45-49 years. Majority of the children had mothers who attained primary level education and the lowest proportion had mothers who attained tertiary level education. Majority of the children lived in rural areas. The largest proportion of children was found in the poorest households with the least proportion of children living in the richest households. Most children had mothers who were non-smokers. More than half of the children had anemia. Majority of the children had mothers who had normal weight at the time of the survey with the least proportion of children having mothers who were obese. The highest proportion of children was found among mothers that had five to fourteen children and the least proportion of children was among mothers that had only one child. Table 1 also shows the prevalence of stunting by socio-demographic characteristic of children. The overall prevalence of stunting among children less than five years was $30 \%$. The distribution of stunting by children's age was uniform across most age groups. The lowest prevalence of stunting was in the age group of 06-10 months. Boys had higher prevalence of stunting than girls, likewise children who had low birth weight ( $<2500$ grams) had her prevalence of stunting. Children whose mothers were living with a partner or were widowed/divorced/separated had high prevalence of stunting. Children in the youngest age group of mothers had the highest prevalence of stunting while those of the oldest mothers (45-49 years) had the least prevalence of stunting. The highest prevalence of stunting was among children whose mothers had no formal education and lowest was in children whose mothers had tertiary level education. Children in rural areas had higher prevalence of stunting than those living in urban areas. Similarly children in poorer and poorest households had the highest prevalence of stunting while those that lived in the richest households had lowest prevalence of stunting. On the same note, children whose mothers use tobacco, had body mass index $<18.5 \mathrm{~kg}$ had high prevalence of stunting and were anemic had high prevalence of stunting. Lastly the distribution of the prevalence of stunting by number of living children was relatively uniform.

Table 2 shows the crude and adjusted odds ratios and their corresponding $95 \%$ confidence interval for stunting among 
children under-five years of age by maternal education level. In the crude model the risk of stunting was highest in children whose mothers had no formal education and lowest in children whose mothers had secondary level education compared to children whose mothers had tertiary level education. When adjusted for confounding variables, the values of the odds ratios reduced but children whose mothers had no formal education still had the highest risk of stunting relative to those whose mothers had either primary or secondary level education.

Table 3 shows the crude and adjusted odds ratios and their corresponding 95\% confidence interval for stunting among children under-five years of age by household wealth index. In the crude model the odds ratios for stunting were similar in children living in the poorest, poorer and middle household wealth index with the lowest risk of stunting observed among children that lived in richer household compared to richest households. In the adjusted model, the values of the odds ratios reduced but remained similar with children in the poorest households having the highest odds ratio for stunting and those in the richer households having the lowest odds ratio for stunting compared to children in the richest households.

Table 1. Characteristics of 3893 (un-weighted 3941) children aged 6-59 months by stunting status.

\begin{tabular}{|c|c|c|c|}
\hline Characteristics & Total n (\%) & Stunted n (\%) & Not stunted n (\%) \\
\hline \multicolumn{4}{|l|}{ Children Age (Months) } \\
\hline $06-10$ & $417(10.70)$ & $069(16.50)$ & $348(83.50)$ \\
\hline $11-15$ & $379(09.70)$ & $109(28.80)$ & $270(71.20)$ \\
\hline $16-20$ & $383(09.80)$ & $146(38.10)$ & $237(61.90)$ \\
\hline $21-25$ & $403(10.40)$ & $155(38.60)$ & $248(61.50)$ \\
\hline $26-30$ & $360(09.20)$ & $128(35.60)$ & $232(64.40)$ \\
\hline $31-35$ & $340(08.70)$ & $116(34.10)$ & $224(65.90)$ \\
\hline $36-40$ & $336(08.60)$ & $114(33.90)$ & $222(66.10)$ \\
\hline $41-45$ & $342(08.80)$ & $112(32.70)$ & $230(67.30)$ \\
\hline $46-50$ & $362(09.30)$ & $083(22.90)$ & $279(77.10)$ \\
\hline $51-55$ & $308(07.90)$ & $080(26.00)$ & $228(74.00)$ \\
\hline $56-59$ & $263(06.80)$ & $058(22.10)$ & $205(77.90)$ \\
\hline Total & $3893(100)$ & $1170(30.10)$ & $2723(69.90)$ \\
\hline \multicolumn{4}{|l|}{ Child`s sex } \\
\hline Male & $1957(50.30)$ & $625(31.90)$ & $1332(68.10)$ \\
\hline Female & $1936(49.70)$ & $545(28.20)$ & $1391(71.80)$ \\
\hline Total & $3893(100)$ & $1170(30.10)$ & $2723(69.90)$ \\
\hline \multicolumn{4}{|l|}{ Child`s birth weight (grams) } \\
\hline$<2500$ & $0240(06.20)$ & $0095(39.60)$ & $0145(60.40)$ \\
\hline$\geq 2500$ & $3653(93.80)$ & $1075(27.60)$ & $2578(70.60)$ \\
\hline Total & $3893(100)$ & $1170(30.10)$ & $2723(69.90)$ \\
\hline \multicolumn{4}{|l|}{ Maternal Marital status } \\
\hline Never in union & $0133(3.40)$ & $34(26.60)$ & $99(74.40)$ \\
\hline Married & $1802(46.30)$ & $493(27.40)$ & $1309(72.60)$ \\
\hline Living with a partner & $1560(40.10)$ & $521(33.40)$ & $1039(66.60)$ \\
\hline Widowed/Divorced/Separated & $0400(10.30)$ & $123(30.80)$ & $277(69.20)$ \\
\hline Total & $3895(100)$ & $1171(30.10)$ & $2724(89.90)$ \\
\hline \multicolumn{4}{|l|}{ Maternal Age (years) } \\
\hline $15-19$ & $180(04.60)$ & $61(33.90)$ & $119(66.10)$ \\
\hline $20-24$ & $978(25.10)$ & $314(32.10)$ & $664(67.90)$ \\
\hline $25-29$ & $1046(26.90)$ & $324(31.00)$ & $722(69.00)$ \\
\hline $30-34$ & $766(19.70)$ & $235(6.00)$ & $531(69.30)$ \\
\hline $35-39$ & $580(14.90)$ & $139(24.00)$ & $441(76.00)$ \\
\hline $40-44$ & $276(7.10)$ & $79(28.60)$ & $197(71.40)$ \\
\hline $45-49$ & $066(1.70)$ & $18(27.30)$ & $48(72.70)$ \\
\hline Total & $3892(100)$ & $1170(30.10)$ & $2722(69.90)$ \\
\hline \multicolumn{4}{|l|}{ Maternal Education Level } \\
\hline No education & $436(11.20)$ & $178(40.80)$ & $258(59.20)$ \\
\hline Primary & $2398(61.60)$ & $765(31.90)$ & $1633(68.10)$ \\
\hline Secondary & $815(29.90)$ & $203(24.90)$ & $612(75.10)$ \\
\hline Tertiary & $244(6.30)$ & $25(10.20)$ & $219(89.80)$ \\
\hline Total & $3893(100)$ & $1171(30.10)$ & $2722(69.90)$ \\
\hline \multicolumn{4}{|l|}{ Type of place of residence } \\
\hline Urban & $815(20.90)$ & $194(23.80)$ & $621(76.20)$ \\
\hline Rural & $3079(79.10)$ & $977(31.70)$ & $2102(68.30)$ \\
\hline Total & $3894(100)$ & $1171(30.10)$ & $2723(69.90)$ \\
\hline \multicolumn{4}{|l|}{ Wealth Index } \\
\hline Poorest & $873(22.40)$ & $303(34.70)$ & $570(65.30)$ \\
\hline Poorer & $803(20.60)$ & $279(34.70)$ & $524(65.30)$ \\
\hline Middle & $769(19.80)$ & $259(33.70)$ & $510(66.30)$ \\
\hline Richer & $681(17.50)$ & $193(28.30)$ & $488(71.70)$ \\
\hline
\end{tabular}




\begin{tabular}{llll}
\hline Characteristics & Total n (\%) & Stunted n (\%) & Not stunted n (\%) \\
\hline $\begin{array}{l}\text { Richest } \\
\text { Total }\end{array}$ & $767(19.70)$ & $136(17.70)$ & $631(82.30)$ \\
Maternal smoking & $3893(100)$ & $1170(30.10)$ & $2723(69.90)$ \\
Yes & & & $35(52.20)$ \\
No & $0067(1.70)$ & $32(47.80)$ & $2687(70.20)$ \\
Total & $3825(98.30)$ & $1138(29.80)$ & $2722(69.90)$ \\
Child anemia & $3892(100)$ & $1170(30.10)$ & $1333(74.60)$ \\
No & $1788(46.20)$ & $455(25.40)$ & $1373(65.90)$ \\
Anemic & $2082(53.80)$ & $709(34.10)$ & $2706(69.90)$ \\
Total & $3870(100)$ & $1164(30.1)$ & $211(64.90)$ \\
Maternal BMI $\left(\mathrm{Kg} / \mathrm{M}^{2}\right)$ & & & $1779(68.10)$ \\
$<18.5$ & $325(8.40)$ & $114(35.10)$ & $498(75.30)$ \\
$18.5-24.99$ & $2612(67.30)$ & $833(31.90)$ & $228(80.30)$ \\
$25-29.99$ & $661(17.00)$ & $163(24.70)$ & $2716(70.00)$ \\
$\geq 30$ & $284(7.30)$ & $56(19.70)$ & \\
Total & $3882(100)$ & $1166(30.00)$ & $357(71.00)$ \\
Number of living children & & & $535(71.80)$ \\
One & $503(12.90)$ & $146(29.00)$ & $480(65.70)$ \\
Two & $745(19.10)$ & $210(28.20)$ & $419(70.60)$ \\
Three & $731(18.80)$ & $251(34.30)$ & $931(70.60)$ \\
Four & $595(15.30)$ & $176(29.60)$ & \\
Five to fourteen & $1319(33.90)$ & $388(29.40)$ & \\
\hline
\end{tabular}

Table 2. Crude and adjusted odds ratios (OR) and their corresponding 95\% confidence interval (CI) for stunting among 3893 (un-weighted 3941$)$ children aged 6-59 months by maternal education level.

\begin{tabular}{lll}
\hline Maternal Education & Crude OR $\mathbf{( 9 5 \%}$ CI) & Adjusted OR (95\% CI) \\
\hline Tertiary & 1.00 & 1.00 \\
No education & $6.04(3.41-10.70)$ & $4.35(2.45-7.71)$ \\
Primary & $4.10(2.40-6.99)$ & $2.74(1.62-4.63)$ \\
Secondary & $2.91(1.63-5.18)$ & $2.30(1.34-3.96)$ \\
\hline
\end{tabular}

Adjusted for household wealth index, children`s age, child sex, size at birth, insurance, maternal education level, maternal body mass index, current maternal marital status, number of living children, place residence.

Table 3. Crude and adjusted odds ratios (OR) and their corresponding 95\% confidence (CI) for stunting among 3893 (un-weighted 3941) children aged 6-59 months by household wealth index.

\begin{tabular}{lll}
\hline Household Wealth Index & Crude OR (95\% CI) & Adjusted OR (95\% CI) \\
\hline Richest & 1.00 & 1.00 \\
Poorest & $2.48(1.87-3.27)$ & $1.78(1.23-2.59)$ \\
Poorer & $2.47(1.81-3.38)$ & $1.88(1.28-2.74)$ \\
Middle & $2.36(1.76-3.17)$ & $1.91(1.31-2.77)$ \\
Richer & $1.84(1.35-2.51)$ & $1.60(1.10-2.32)$ \\
\hline
\end{tabular}

Adjusted for children`s age, child sex, size at birth, insured, maternal education level, maternal body mass index, current marital status, number of living children, type place residence.

\section{Discussion}

In this study, we used two measures of socio-economic status to assess socio-economic inequalities in stunting among children under-five years of age in Uganda. As shown in the adjusted model in Table 2, maternal formal education was associated with stunting among children under-five years of age. Our findings therefore indicate existing socioeconomic inequalities in stunting at the time of conduct of the 2016 UDHS. The risk of stunting tended to decrease with increase in the level of maternal formal education. Children born to mothers who had no formal education were found to have three-fold increase in the risk of stunting compared to those whose mothers had tertiary level formal education. Likewise children whose mothers had primary and secondary level education were 1.74 and 1.30 times more likely be stunted compared to those whose mothers had tertiary level education respectively. These results are in agreement with those of a Ugandan cross-sectional study based on 2011 Uganda Demographic and Health Survey data which found an association between maternal formal education level and stunting among children under-five years of age [16] and also similar to the findings of another Ugandan study that used data from four Uganda Demographic and health surveys preceding the 2016 UDHS [8]. However the results of this study are not in agreement with those of a Ugandan crosssectional study [34] conducted in in Nakasongola and Nakaseke districts in central Uganda that found an increasing risk of stunting by maternal education level (children whose mothers had primary or no education were less likely to be stunted. Perhaps, it's because the later study was conducted 
in a localized area and had smaller sample size, let alone the cross-sectional nature of the study design where reverse causality cannot be ruled out. Despite of some inconsistency with the findings of other previous studies, the findings of this study showed existence of inequalities in stunting among children under-five years of age by maternal education level. This might be linked to the possibility that mothers with no or low level education might have inadequate knowledge on nutrition, infant and young child feeding practices, and inability to afford a wide variety of nutritious food and timely high quality healthcare services for their children.

When inequality in stunting among children under-five years of age was assessed using, household wealth index, a measure widely used in developing countries for assessing standard of living in households, the results showed that household socio-economic status is an important predictor of stunting. The risk of stunting among children under-five years of age was $78 \%, 88 \%, 91 \%$ and $60 \%$ higher among children in the poorest, poorer, middle and richer households compared to those in the richest households. There seems to be an increasing trend in the risk of stunting among children under five years by increasing household wealth level, however this trend isn't clear as a relatively low risk of stunting was observed among children that lived in the richer households compared to the richest households. Perhaps this points to residual confounding or misclassification of outcome with respect to exposure and vice-versa. These findings are in agreement with the results of cross-sectional studies conducted in Uganda [8, 16] and in other developing countries [35, 36].

\section{Limitations and Strengths}

The cross-sectional nature of the study limits causal inference. Results might have also been influenced by unknown confounders not adjusted for in the analysis. Despite of these limitations, the results of this study can be generalized to all Ugandan children under-five years of age in the general population and adds into the limited literature on social inequalities in stunting among children under-five years of age in Uganda.

\section{Conclusions}

Socio-economic inequality in stunting among children under-five years of age still persists in Uganda. An inverse relationship was found between maternal, household socioeconomic status and stunting among children aged 6-59 months in Uganda. Therefore it might be important to target stunting prevention interventions to less affluent motherchild dyads and families if social disparities in the burden of stunting among children less 5 five years of age are to be reduced in Uganda.

\section{Conflict of Interest}

This study was conducted without funding from any entity or individual. Therefore the authors have no conflict of interest to declare.

\section{Acknowledgements}

We acknowledge the management of Victoria University for providing suitable environment for conduct of scientific research.

\section{References}

[1] Black, R. E., et al., Maternal and child undernutrition: global and regional exposures and health consequences. The lancet, 2008. 371 (9608): p. 243-260.

[2] Dewey, K. G. and K. Begum, Long - term consequences of stunting in early life. Maternal \& child nutrition, 2011. 7: p. 518 .

[3] Stunting, C., Context, Causes and Consequences WHO Conceptual Framework. 2013, WHO.

[4] Black, R. E., et al., Maternal and child undernutrition and overweight in low-income and middle-income countries. The lancet, 2013. 382 (9890): p. 427-451.

[5] De Onis, M., M. Blössner, and E. Borghi, Prevalence and trends of stunting among pre-school children, 1990-2020. Public health nutrition, 2012. 15 (1): p. 142-148.

[6] UNICEF, Joint child malnutrition estimates-Levels and trends. 2017.

[7] UNICEF, W., and The World Bank., Levels and trends in child malnutrition: key findings of the 2019 Edition of the Joint Child Malnutrition Estimates. 2019: Geneva.

[8] Yang, Y. Y., et al., Trends and determinants of stunting among under-5s: evidence from the 1995, 2001, 2006 and 2011 Uganda Demographic and Health Surveys. Public health nutrition, 2018. 21 (16): p. 2915-2928.

[9] Statistics, U. B. o. and U. ICF \% J Kampala, Uganda demographic and health survey 2016: key indicators report. 2017, UBOS, and Rockville Maryland.

[10] Bank, W., the Uganda Poverty Assessment Report 20162016.

[11] Statistics, U. B. o. and ICF, Uganda Demographic and Health Survey 2016: Key Indicators Report. 2017, Uganda Bureau of Statistics (UBOS), and Rockville, MD: UBOS and ICF Kampala.

[12] Nambuusi, B. B., et al., The effects and contribution of childhood diseases on the geographical distribution of allcause under-five mortality in Uganda. Parasite Epidemiology and Control, 2019: p. e00089.

[13] Achadi, E., et al., Global Nutrition Report: From Promise to Impact: Ending Malnutrition by 2030. 2016: International Food Policy Research Institute.

[14] Marmot, M., et al., Fair society, healthy lives. 2008.

[15] Adekanmbi, V. T., G. A. Kayode, and O. A. Uthman, Individual and contextual factors associated with childhood stunting in Nigeria: a multilevel analysis. Maternal \& child nutrition, 2013.9 (2): p. 244-259.

[16] Mawa, R. and S. Lawoko, Malnutrition Among Children Under Five Years in Uganda. American Journal of Health Research, 2018. 6 (2): p. 56-66. 
[17] Dake, S. K., et al., Predictors of stunting among children 6-59 months of age in Sodo Zuria District, South Ethiopia: a community based cross-sectional study. 2019. 5 (1): p. 23.

[18] Akombi, B., et al., Stunting, wasting and underweight in subSaharan Africa: a systematic review. 2017. 14 (8): p. 863.

[19] Casale, D., G. Espi, and S. A. J. P. h. n. Norris, Estimating the pathways through which maternal education affects stunting: evidence from an urban cohort in South Africa. 2018. 21 (10): p. $1810-1818$.

[20] Mawa, R. and S. J. A. J. o. H. R. Lawoko, Malnutrition Among Children Under Five Years in Uganda. 2018. 6 (2): p. 56-66.

[21] Abuya, B., et al., Influence of maternal education on child immunization and stunting in Kenya. 2011. 15 (8): p. 13891399.

[22] Keino, S., et al., Determinants of stunting and overweight among young children and adolescents in sub-Saharan Africa. 2014. 35 (2): p. 167-178.

[23] Howe, L. D., J. R. Hargreaves, and S. R. Huttly, Issues in the construction of wealth indices for the measurement of socioeconomic position in low-income countries. Emerging themes in epidemiology, 2008. 5 (1): p. 3.

[24] Mensch, B. S., et al., Evidence for causal links between education and maternal and child health: Systematic review. Tropical Medicine \& International Health, 2019.

[25] Habaasa, G. J. B. p., An investigation on factors associated with malnutrition among underfive children in Nakaseke and Nakasongola districts, Uganda. 2015. 15 (1): p. 134.

[26] Kikafunda, J. and J. Tumwine, Diet and socio-economic factors and their association with the nutritional status of preschool children in a low income suburb of Kampala City, Uganda. East African medical journal, 2006. 83 (10): p. 565574.
[27] Prentice, A. M. J. N. and G. Yearbook, Stunting in Developing Countries. 2019. 119: p. 171-183.

[28] Wamani, H., et al., Boys are more stunted than girls in subSaharan Africa: a meta-analysis of 16 demographic and health surveys. 2007.7 (1): p. 17.

[29] Schoenbuchner, S. M., et al., The relationship between wasting and stunting: a retrospective cohort analysis of longitudinal data in Gambian children from 1976 to 2016. The American journal of clinical nutrition, 2019.

[30] Foundation, N., The HUNGaMA Survey Report 2011.

[31] Abuya, B. A., J. Ciera, and E. J. B. p. Kimani-Murage, Effect of mother's education on child's nutritional status in the slums of Nairobi. 2012. 12 (1): p. 80.

[32] Fikadu, T., S. Assegid, and L. Dube, Factors associated with stunting among children of age 24 to 59 months in Meskan district, Gurage Zone, South Ethiopia: a case-control study. BMC Public Health, 2014. 14 (1): p. 800.

[33] Jolliffe, I. T. and J. Cadima, Principal component analysis: a review and recent developments. Philosophical Transactions of the Royal Society A: Mathematical, Physical and Engineering Sciences, 2016. 374 (2065): p. 20150202.

[34] Habaasa, G., An investigation on factors associated with malnutrition among underfive children in Nakaseke and Nakasongola districts, Uganda. BMC pediatrics, 2015. 15 (1): p. 134.

[35] Mistry, S. K., et al., Individual-, maternal-and household-level factors associated with stunting among children aged 0-23 months in Bangladesh. Public health nutrition, 2019: p. 1-10.

[36] Sharaf, M. F., E. I. Mansour, and A. S. Rashad, child nutritional status in egypt: a comprehensive analysis of socioeconomic determinants using a quantile regression approach. Journal of biosocial science, 2019.51 (1): p. 1-17. 